\title{
3 Research Square \\ Seizure Detection and Tracking Through Portable Technologies: Protocol for a Systematic Review
}

Elisabeth Beauchamp-Chalifour

Universite Laval https://orcid.org/0000-0002-2854-8073

Marie-Pierre Gagnon ( $\triangle$ Marie-Pierre.Gagnon@fsi.ulaval.ca )

Universite Laval

Tamara Herrera Fortin

Universite de Montreal

Elie Bou Assi

Universite de Montreal

Dang Khoa Nguyen

Universite de Montreal

\section{Protocol}

Keywords: Epilepsy, SUDEP, Seizures, Detection, Tracking, Portable technologies, Evaluation

Posted Date: February 10th, 2021

DOI: https://doi.org/10.21203/rs.3.rs-66216/v2

License: (c) (1) This work is licensed under a Creative Commons Attribution 4.0 International License.

Read Full License 


\section{Abstract}

\section{Background}

Epilepsy is a chronic neurological disease characterized by recurrent seizures due to excessive neuronal discharges and abnormal neuronal discharges. $30-40 \%$ of them are pharmaco-resistant, which is associated with cognitive impairment. seizure-related injuries and a higher risk of sudden unexpected death in epilepsy (SUDEP). The only way to minimize the risk of SUDEP is having a control of seizures. However, epileptics living or get a seizure alone can't report the exact information to their physicians. Then, the level of gravity of their disease is inexact. Currently, the only way to identify a seizure is by electroencephalography (EEG), used by healthcare resources. It requires medical staff for its installation and interpretation. Nonetheless, a lot of new technologies developed for recording seizures at home have been developed. These one hasn't optimally included epileptics and only a few went to a clinical trial; thus, scientific evidence is limited regarding their effectiveness.

\section{Methods}

The methodological approach is based in the Cochrane handbook for conducting systematic review. All studies in any languages about the technologies detecting seizures will be compared for any type of epilepsy and for any outcome measured. Two reviewers will independently screen the results for MEDLINE [PubMed, 1951-June 2019], EMBASE [OVID, 1947-June 2019], the Cochrane Register of Controlled Trails (CENTRAL) [up to June 2019] and grey literature [up to June 2019]. Extracted data will be recorded, categorized and summarized.

\section{Discussion}

The expected results would be the too short time of the trials and many positive false to get a conclusion of the effectiveness of the technologies studied. This systematic review is needed in order to improve knowledge of the effectiveness of technologies for detecting seizures and use this information for the development of a personalized tool for detecting seizures on the basis of the best available scientific evidence.

\section{Systematic review registration}

PROSPERO CRD42020129787.

\section{Introduction}

\subsection{Context}

Epilepsy is a chronic disease characterized by recurrent seizures resulting from excessive and abnormal neuronal discharges. There are currently over 50 million people worldwide affected by this medical condition. It is estimated that by 2031,415800 Canadians would be affected [1]. Common causes of 
epilepsy include perinatal injury, malformations of cortical development, genetic mutations, scarring from prior brain insults (e.g., stroke, infection and trauma), tumors, and degenerative disorders. While the first line of treatment consists of chronic anticonvulsivant medication therapy, 30-40\% of patients are pharmaco-resistant [2]. The International League Against Epilepsy (ILAE) defines pharmaco-resistant epilepsy as failure to achieve seizure freedom following adequate trials of two tolerated and appropriately chosen antiepileptic drugs [3]. Uncontrolled seizures are associated with significant physical, social, and psychological impairment. Moreover, a major risk of uncontrolled epilepsy is premature death, either from seizure-related injury or sudden unexpected death in epilepsy (SUDEP). In the United States alone, 2,000 SUDEP occur each year [2]. Patients with epilepsy living alone and patients experiencing nocturnal seizures are at a higher risk of SUDEP as seizures may go unnoticed and hence underreported to their physicians.

Hospitalization is the common way to diagnose and monitor epileptic seizures using video combined with electroencephalogram (video-EEG) [4]. Such inpatient video-EEG monitoring studies are performed for specific indications (ex. To confirm the epileptic nature of atypical spells, to manage acute exacerbation of seizures, to localize the onset of seizures in the context of epilepsy surgery), are of relatively short duration (few days to 2 weeks), and are costly. Ambulatory portable video-EEG systems are available, but recordings are frequently contaminated by movement artifacts. Obviously, scalp electrodes are not meant for prolonged (weeks, months, years) use both for comfort and esthetic reasons. In the last ten years, many non-obtrusive seizure detection devices, and electronic applications for recording seizures have been developed such as smart watches, smart clothing, tactile mattresses, accelerometers, microphones or cameras [5]. Indeed, during seizures, patients present changes in oxygen saturation [6], heart rate [7], breathing rate [8] and body fluid movements [9], all of which can be monitored by non-obstructive devices. Non-obtrusive home-based or ambulatory seizure detection systems could be useful for providing a more accurate assessment of seizure frequency. In addition, to improve epilepsy management and reducing epilepsy-related costs, such seizure detection systems could trigger alarms with the potential to prevent or minimize seizure-related injuries or even death.

Efficient and reliable seizure detection with such non-obtrusive devices is likely to be beneficial to patients with regard to safety, treatment, and quality of life (QoL). Unfortunately, many of these devices have not optimally validated either inside the hospital (in the epilepsy monitoring unit with the gold standard i.e. video-EEG) or outside the hospital to assess its true ambulatory value, on large groups of patients, and by integrating the viewpoints of epileptic patients [5]. In a systematic review published in 2016, Jory et al. (2016) [10] concluded that detection technologies still involved high rates of false positives. Since 2016, there has been a renewed interest in such non-obstrusive seizure detection devices and further technological advancements have been made. In this systematic review, we will therefore include new evidence from studies published since the previous review [10] while assessing the effectiveness of proposed technologies and their impact on the QoL of patients.

\subsection{Objectives}


The global aim of this study is to perform a systematic review and environmental scan of proposed solutions for the detection of epileptic seizures and assess their reliability, performance, safety, acceptability, validity and impact on patient behavior. Secondary objectives include assessing the factors that could influence the adoption of these technologies. The primary outcomes will focus on healthrelated quality of life (HRQoL) and other important aspects of patient outcomes (e.g. acceptance, satisfaction of the e-Health solutions).

\subsection{Review questions}

1) Which smart portable solutions are currently available for detecting seizures?

2) What are the needs, perceptions and attitude of people living with any type of epilepsy, their informal caregivers and the healthcare professionals towards smart portable solutions for detecting epileptic seizures?

3) What are the related motivators, barriers, challenges and moderating factors experienced by these individuals towards the adoption of the smart portable solutions?

\section{Methods}

This mixed-method systematic review protocol is designed based on PRISMA-P statement [11] and follows the methodological approach proposed in the Cochrane handbook for conducting systematic reviews [12].

\subsection{Eligibility criteria}

Iype of studies: All empirical studies (quantitative, qualitative, mixed methods) on the proposed devices for the detection of any type of seizures (focal or generalized; motor or non-motor; unknown) for any outcome measured (e.g., validity for seizure detection, number of seizures, patient satisfaction, etc.) will be considered. All studies from database creation up to 2019 will be included. All languages will be included. Exclusion criteria: editorials, comments, letters to the editor and technical memo. Conditions or domains being studied: This systematic review is positioned in the field of epilepsy, seizures, epilepsy treatment and management, effect on QoL of epilepsy, and the prevention of seizure-related adverse events through technology. Its main purpose is to identify currently available solutions.

This systematic review also focuses on the needs, perceptions and attitudes towards the use of portable technologies for tracking seizures in people living with epilepsy, their professionals or informal caregivers and healthcare professionals. Moreover, the review will identify the perceived motivators and factors influencing the use or non-use of these technologies. Type of participants: Patients with a diagnostic of epilepsy (every type). Iype of interventions: Studies assessing any type of portable technology used for detecting epilepsy seizures. Comparator(s)/control: The reference group will not have any intervention or will get other methods for detecting seizures (not based on information and communication technologies, e.g., standard EEG). 


\subsection{Information sources}

We developed a search strategy that will be used for MEDLINE [PubMed, 1951-June 2019], EMBASE [OVID, 1947-June 2019] and the Cochrane Register of Controlled Trails (CENTRAL) [up to June 2019]. We will identify randomized controlled trials by validated filters for MEDLINE and EMBASE. We will conduct similar research in Web of Science and CINAHL. We will also consider grey literature and ongoing studies by searching Google and specialized sites on epilepsy.

\subsection{Search strategy (see Appendix 1)}

The MEDLINE strategy is presented in Appendix 1. A similar search will be conducted with EMBASE, CENTRAL, The Cochrane Library, CINAHL, and the Web of Science.

\subsection{Study records}

\subsubsection{Data management}

Records and data will be exported to Rayyan [13] directly from databases for the selection process.

\subsubsection{Selection process}

The selection process will be done by two authors independently through each phase of the review (screening, eligibility and inclusion in meta-analysis). We will merge search results by using EndNote X8 and remove duplicate records of the same report. First, we will examine titles and remove obviously irrelevant reports. Second, we will review abstracts and we will retrieve full text of the potentially relevant reports. We will examine full-text reports for compliance of studies with eligibility criteria. We will review the selected studies and make final decisions on inclusion and proceed to data extraction.

\subsubsection{Data collection process}

We will create a data abstraction form using Excel to standardize the data collection process, which will be pilot tested. Data will be collected independently by the same two authors previously identified. We will compare the results of both data extraction. Electronic searches for text will aid to locate useful data. A translator will be contacted for studies published in languages other than English or French.

\subsection{Data items}

We will gather: (1) characteristics of the studies selected (year, author, country, design, method and follow-up); (2) Demographic data of the population included in the studies assessed (age, diagnosis, sex, comorbidities); (3) Individual information (type of epilepsy, hospitalization time, e-Health solutions used, number of seizures, performance assessment (sensitivity, specificity). We will present the results in a narrative form.

\subsection{Outcomes and prioritization}


Prioritized outcomes of this systematic review are the effects of the technologies on the health and the quality of life of the patients with epilepsy. We will also take into account the validity of the measures, the number of hospitalisations due to relapse, the duration of hospital stays, and costs related to the intervention.

The secondary outcomes will include knowledge of e-Health solutions and health behaviors of all participant groups and perceived social support specifically for the epileptic participant group. We will also include the perceptions and needs of all participant groups regarding e-Health solutions, as well as the perceived motivators and barriers for use or non-use of e-Health solutions in all participant groups. Comparing the performance of various devices will be another secondary outcome.

\subsection{Risk of bias in individual studies}

The risk of bias will be assessed independently by the two reviewers. At the study level, we use the Cochrane Collaboration's Tool for assessing risk of bias (RoB) for randomized controlled trial (RCT). We will use the Risk of Bias in Non-randomized Studies of interventions (ROBINS-I) tool from Cochrane to evaluate the presence of bias in non-randomized trials, the CERQUAL for qualitative studies and the MMAT for the mixed-methods. Although blinding will likely not be possible as participants and providers will know what technology they are using, data analysts should be blinded to the intervention. Attention will be put towards intervention, selection and attrition bias.

\subsection{Data Synthesis}

Two independent reviewers will analyze data from included studies in Review Manager (Rev-Man) version 5.3. Summary data for each intervention group will be separately collected and entered in Rev-Man. Mean differences (MD) and standardized mean differences (SMD) with $95 \%$ confidence intervals will be used to compare scores. Cochran-Mantel-Haenszel method will be used for dichotomous variables ${ }^{\text {[12]. Subgroup }}$ analysis will include type of technology used (e.g. smart watches, smart clothing, tactile mattresses, accelerometers, microphones and cameras).

To answer to the second and third question of this systematic review, we will use a qualitative thematic analysis [14]. The two reviewers will extract the main themes as reported by the study author and other significative information. These main themes will be grouped into lower and higher order themes. We will also qualify the quantitative results by a narrative approach and integrate results of the qualitative and quantitative studies through a convergent approach.

\section{Clinical Application Of Results}

A clear understanding of these issues could potentially better guide neurologists specialized in epilepsy in deciding whether to recommend the use of smart watches, smart clothing, tactile mattresses, accelerometers, microphones and cameras or any combination of these technologies to their patients. Other healthcare personal (e.g. primary care team) will be able to understand the role of technology in 
epilepsy management and use it properly. Patients and informal caregivers will be able to make informed decisions regarding the use of such technologies. Finally, a better understanding of patients' needs and preferences regarding seizure detection might provide guidance for future developments in this field.

\section{List Of Abbreviations}

EEG: Electroencephalography

HRQoL: Health-related quality of life

ILAE: International League Against Epilepsy

MD: Mean differences

PRISMA.Preferred Reporting Items for Systematic Reviews and Meta-Analyses

QoL: Quality of life

RCT: Randomized controlled trial

RoB : Risk of bias

ROBINS-I: Risk of Bias in Non-randomized Studies of interventions

SMD: Standardized mean differences

SUDEP: Sudden unexpected death in epilepsy

Video-EEG: Video combined with electroencephalogram

\section{Declarations}

\section{Ethics declarations}

\section{Ethics approval and consent to participate}

This study did not require approval from an ethics committee. No human subjects are involved in this study.

\section{Consent for publication}

The authors declare that they have no individual person's data in any form used.

\section{Competing interests}

The authors declare that they have no competing interests. 


\section{Publisher's Note}

Springer Nature remains neutral with regard to jurisdictional claims in published maps and institutional affiliations.

\section{Acknowledgements}

A special thanks to our partners from the CHUM's Research Center with their epilepsy's expertise.

\section{Funding}

This work received CIHR-NSERC CHRP program funding. The reference number is CPG-163983.

\section{Availability of data and materials}

All data generated and analyzed during this study will be included in the published article.

\section{Author information}

\section{Contributions}

EBC and MPG conceived the idea for this review. EBC drafted the protocol under the supervision of MPG, DKN, EBA and THF. All authors read and approved the final manuscript. Neither the funding body, sponsor nor institution had any involvement in the development of this protocol.

\section{References}

1. Public Health Agency of Canada., Mapping Connections: An understanding of neurological conditions in Canada, in Chapter 3: Mapping Connections: An understanding of neurological conditions in Canada - Scope (prevalence and incidence). 2014, Canada Government.

2. Laxer, K.D., et al., The consequences of refractory epilepsy and its treatment. Epilepsy \& Behavior, 2014. 37: p. 59-70.

3. Kwan, P., et al., Definition of drug resistant epilepsy: consensus proposal by the ad hoc Task Force of the ILAE Commission on Therapeutic Strategies. Epilepsia, 2010. 51(6): p. 1069-77.

4. Hampel, K.G., et al., [Diagnostic challenges in epilepsy]. Rev Neurol, 2019. 68(6): p. 255-263.

5. Ramgopal, S., et al., Seizure detection, seizure prediction, and closed-loop warning systems in epilepsy. Epilepsy Behav, 2014. 37: p. 291-307.

6. Jaychandran, R., et al., Monitoring peri-ictal changes in heart rate variability, oxygen saturation and blood pressure in epilepsy monitoring unit. Epilepsy Res, 2016. 125: p. 10-8.

7. Bruno, E., A. Biondi, and M.P. Richardson, Pre-ictal heart rate changes: A systematic review and metaanalysis. Seizure, 2018. 55: p. 48-56. 
8. Barot, N. and M. Nei, Autonomic aspects of sudden unexpected death in epilepsy (SUDEP). Clin Auton Res, 2019. 29(2): p. 151-160.

9. Bromfield EB, C.J., Sirven JI, editors.,, An Introduction to Epilepsy [Internet]. . Chapter 2, Clinical Epilepsy. . 2006, West Hartford (CT): American Epilepsy Society.

10. Jory, C., et al., Safe and sound? A systematic literature review of seizure detection methods for personal use. Seizure, 2016. 36: p. 4-15.

11. Moher, D., et al., Preferred reporting items for systematic review and meta-analysis protocols (PRISMA-P) 2015 statement. Systematic Reviews, 2015. 4(1): p. 1.

12. Higgins, J.P.T., et al., Cochrane Handbook for Systematic Reviews of Interventions. 2019: Wiley.

13. Ouzzani, M., et al., Rayyan-a web and mobile app for systematic reviews. Systematic Reviews, 2016. 5(1): p. 210.

\section{Supplementary Files}

This is a list of supplementary files associated with this preprint. Click to download.

- APPENDIX1.docX

- PRISMA2009checklist.doc 